Crise(s) dans le monde ibérique et ibéro-américain

\title{
Los muertos (1947) de José Luis Hidalgo et la crise de la poésie dans la première posguerra espagnole : le « double-entendre » d'une poésie existentielle pour dire l'angoisse d'une époque et d'un pays
}

\section{Emmanuel Le Vagueresse}

\section{(2) OpenEdition}

\section{Journals}

Édition électronique

URL : https://journals.openedition.org/cher/3720

DOI : $10.4000 /$ cher.3720

ISSN : 2803-5992

Éditeur

Presses universitaires de Strasbourg

Édition imprimée

Date de publication : 1 décembre 2015

Pagination : 149-161

ISBN : 978-2-86820-913-9

ISSN : 1968-035X

Référence électronique

Emmanuel Le Vagueresse, «Los muertos (1947) de José Luis Hidalgo et la crise de la poésie dans la première posguerra espagnole : le « double-entendre » d'une poésie existentielle pour dire l'angoisse d'une époque et d'un pays », reCHERches [En ligne], 15 | 2015, mis en ligne le 01 décembre 2021, consulté le 20 décembre 2021. URL : http://journals.openedition.org/cher/3720 ; DOI : https://doi.org/ 10.4000/cher.3720

\section{(c) (i)(3)}

Ce(tte) œuvre est mise à disposition selon les termes de la Licence Creative Commons Attribution -

Pas d'Utilisation Commerciale - Partage dans les Mêmes Conditions 4.0 International. 


\title{
Los muertos (1947) de José Luis Hidalgo et la crise de la poésie dans la première posguerra espagnole: le "double-entendre » d'une poésie existentielle pour dire l'angoisse d'une époque et d'un pays
}

\author{
Emmanuel Le Vagueresse \\ Université de Reims Champagne-Ardenne \\ CIRLEP EA-4299
}

\begin{abstract}
Tous aimerions montrer comment la poésie de José Luis Hidalgo (19191947), poète espagnol, mais aussi peintre, qui fit un passage éclair dans le paysage littéraire espagnol de la posguerra, tout en le marquant profondément (Raíz, 1944, Los animales, 1945 et surtout Los muertos, 1947 [posth.], recueil sur lequel nous nous appuierons), s'inscrit dans la problématique de la crise poétique espagnole de l'après-guerre civile et mondiale, à une époque où les écrivains ne peuvent plus écrire comme "avant», c'est-à-dire dans les années d'avantguerre(s), soit, pour l'Espagne, comme feu les esthétisants «Poètes de 27 ».

La poétique d'un José Luis Hidalgo - très peu étudiée encore en Francel, mais beaucoup en Espagne -, ce poète cantabre mort avant ses 30 ans de la tuberculose, dut composer avec la censure du premier franquisme, ces effroyables années quarante de glaciation idéologique, mais aussi esthétique et culturelle. Et, ce, malgré la présence d'une revue comme Proel (Santander), qui le publia, tout comme elle publia le «local» José Hierro ou Vicente Aleixandre. Nous verrons alors quelles sont les stratégies choisies par le poète pour dire et son angoisse existentielle d'un monde où Dieu se fait pour le moins attendre et son mal-être d'Espagnol, à un moment où l'existentialisme athée et ses doutes étaient autant proscrits que la critique politique ou idéologique, dans l'Espagne des Vainqueurs très chrétiens.
\end{abstract}

1 À l'exception notable des travaux de Lidio Jesús Fernández, qui y a consacré plusieurs articles et, surtout, sa Thèse de $3^{e}$ cycle (Fernández 1983).

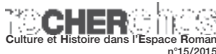


On verra aussi comment cette crise métaphysique de Hidalgo se double d'une crise de sa capacité même à s'exprimer, avec cette épée de Damoclès de la censure (et aussi, difficile à évaluer, de la perverse autocensure) qui pèse sur lui, comme sur d'autres poètes, simples «soldats de mots» (Blaise Cendrars). S'il n'est absolument pas question de refuser toute origine personnelle et intime à cette crise, chez quelqu'un qui s'est su condamné très tôt par la maladie et a eu une prescience précoce de sa propre mort, on préférera insister sur l'atmosphère de l'époque, dans l'Espagne franquiste, pour expliquer la motivation de ces poèmes, conjugaisons, au final, d'un malaise à la fois intime et collectif ${ }^{2}$. Seul importera ici de se pencher sur la traduction esthétique/formelle de cette peine, de ce désespoir, de cette crise, en poème.

D’un mot, on rappellera que les années quarante sont celles de la plus terrible posguerra espagnole. Dans ce contexte, quelques productions surnagent - on ne pense ici qu’aux écrivains qui sont restés en Espagne sans être expressément du côté des "Vainqueurs» nationalistes -, pour la poésie, par exemple, les désormais célèbres Sombra del paraíso de Vicente Aleixandre et Hijos de la ira de Dámaso Alonso, tous deux en 1944, deux recueils qui redonnèrent un peu d'espoir (poétique) aux écrivains ou lecteurs espagnols ${ }^{3}$.

Ces recueils de poésie emplis de suggestion(s) s'inscrivent alors dans ce que l'on appelle en castillan la poésie «desarraigada», par rapport à celle qui surgira un peu avant le mitan des années cinquante, plus «enracinée» dans le social, voire le politique avec la très légère ouverture de l'Espagne au monde extérieur, une poésie qui s'exprimera, quant à elle, avec une extrême prudence tout en défiant les censeurs, et dont un Gabriel Celaya ou un Blas de Otero seront les

2 Interaction entre individu et société, personne et histoire, que les arts ont toujours connue (voir les souffrances des Romantiques, les dénonciations des Réalistes, les désirs d'un autre monde parallèle des Symbolistes, et un long etc.) et qu'il est malaisé - mais peut-être vain? - de débrouiller. Malgré tout, on ne veut pas réduire non plus une individualité à une génération d'artistes ou d'écrivains, attitude contre laquelle Juan Goytisolo nous met bien en garde, parlant du « [...] rutinario comodín generacional, esto es, el agrupamiento de los creadores en funcción de su edad que borra la individualidad del novelista o poeta respecto a sus coetáneos [...]» (Goytisolo 2012: 62). Sur les mécanismes difficiles à mesurer de l'influence d'une "atmosphère" spatio-temporelle sur la création, voir les pages éclairantes de Pascal Ory dans son ouvrage de référence L'histoire culturelle, particulièrement le sous-chapitre "La culture détermine aussi la culture» (Ory 2011: 73-78) - qui se penche aussi sur la validité de la notion de "génération» - et le chapitre «Formes» dans son entier (Ory 2011: 96-116). Voir aussi, pour une apologie plus spécifique des influences chez les artistes, la conférence d'André Gide datant de 1900, De l'influence en littérature (Gide: 2010).

3 Et dans une moindre mesure: Arcángel de mi noche de Vicente Gaos (1943), Los años de Julio Maruri (1944), Tierra sin nosotros de José Hierro (1947), les deux grands amis de Hidalgo. Il y eut aussi des revues poétiques, créées à l'époque, comme Corcel (1942-1949, à Valence), Espadaña (1944-1951, à León) et Proel, citée plus haut, fondée à Santander en 1944 (avec une parution jusqu'en 1950) pour contrer la poésie néogarcilasiste aux relents souvent franquistes de la revue Garcilaso (1943-1946, à Madrid). 
symboles. Mais en 1947, on se situe encore dans lécriture d'un questionnement qui semble flou, abstrait, cérébral et existentiel.

Pour autant, cette poésie est fortement liée, on l’a vu, à une époque, et pour les écrivains (ici, poètes) espagnols nés aux alentours de 1920, comme Hidalgo, Julio Maruri ou José Hierro, elle est liée à cette guerre qu'ils n’ont pas connue en tant que combattants - car ils étaient trop jeunes et dont la blessure, qu'ils n'auraient même pas reçue, contrairement à leurs aînés immédiats les hante.

Quant à José Luis Hidalgo, il propose avec son recueil Los muertos un ensemble de poèmes "de crise», non seulement dans le sens de: "phase grave dans l'évolution des choses, des événements, des idées", mais aussi dans le sens médical - d'ailleurs premier dans l'ordre des acceptions du mot, chronologiquement parlant - de: «moment d'une maladie caractérisé par un changement subit et généralement décisif, en bien ou en mal $»^{4}$, une possibilité «en bien» - dont nous nous souviendrons en conclusion.

Dans ce recueil écrit entre 1944 et 1945 et dont les épreuves furent corrigées sur son lit d'agonie, le poète tente de conjuguer le dire d'une crise à la fois personnelle et collective, tout en prenant garde à ne pas s'affronter directement à la censure, qui constitue elle-même un élément de cette crise (la censure dans son état généralisé et extrême procède en effet d'un pays autoritaire, sinon dictatorial) aux traductions poétiques certaines, puisquelle empêche une expression franche de ces problèmes intimes - impossibilité d'évoquer, par exemple, le suicide et/ou nationaux - impossibilité de montrer une image sombre du pays.

D’où une stratégie d'expression "oblique» qui, pour dire le mal-être général d'un peuple, passe par un mal-être personnel, ou, à l'inverse, dit un mal-être si général qu'il en devient métaphysique et non plus social ou politique, bien que le lectorat ne soit pas dupe ${ }^{5}$.

Dès le titre, funèbre à souhait ${ }^{6}$, dès les deux exergues empruntés à - et traduits en castillan de - Michel-Ange et Goethe ${ }^{7}$, le poète donne le ton programmatique

4 Ces deux définitions proviennent du Nouveau Petit Robert, éd. de 2013.

5 Voir notre étude: «Lire en Espagne sous Franco: l'heure du lecteur», à lire dans le volume coordonné par Louise Bénat-Tachot et Jean Vilar (Bénat-Tachot/ Vilar 2004: 241-251). Quant au peu de capacité, réelle ou supposée, de certains censeurs à décrypter parfois les doubles sens, elle reste à la fois une énigme et l'objet de possibles explications sur lesquelles nous ne pouvons pas nous attarder ici (faible formation à l'exégèse littéraire, impossibilité à tout censurer, relâchement ou indulgence dans la tâche, désir d'afficher une certaine "ouverture», etc.). La poésie était également moins exposée que d'autres formes d'expression (chanson, théâtre, presse...) du fait de son petit nombre de lecteurs, donc de sa relative innocuité pour le régime franquiste.

6 José Luis Hidalgo, Los muertos (Hidalgo 2000: 95-157). Nous citons les poèmes de Los muertos d'après la belle édition récente des Poesías completas de Hidalgo par le poète Juan Antonio González Fuentes (il y en a eu plusieurs autres par le passé). On donnera simplement le numéro des pages entre parenthèses après chaque dernier vers ou groupe de vers du poème cité.

7 Respectivement: «No nace pensamiento en mí / que no lleve esculpida la muerte» (source identifiée par nos soins: lettre à Giorgio Vasari, 1555) et «Cuando mi alma 
du livre, c'est-à-dire à la fois son obsession de la mort et sa propre quête, à l'orée de sa propre mort, d' «un» Dieu - malgré tout restitué avec sa majuscule, peutêtre pour complaire à la censure nationale-catholique - qui l'écouterait. Ce qui fait du locuteur poétique d'emprunt tout sauf un homme à la foi chevillée au corps, plutôt un converti «de la dernière heure", au sens strict de l'expression.

Parmi les 56 poèmes qui forment ce recueil, divisé en quatre parties ${ }^{8}$, la totalité de ceux-ci développe cette idée de mort(s) qui rôde(nt), soit celle à venir du locuteur poétique, le double - jusquà un certain point - du poète, soit celles des êtres disparus, les «muertos» du titre, écho implicite, vu les silences nécessaires de l'époque en Espagne, des morts de la toute récente guerre civile?. La quasi-totalité des titres évoquent d'ailleurs dans leur isotopie soit la mort (via ses polyptotes), soit la souffrance, la tristesse, la solitude, soit encore le doute, l'interrogation, la quête, en tant que champs sémantiques et lexicaux.

Le premier poème, par exemple, intitulé fort à propos «Silencio», se réfère en effet à la fois au silence de la mort et/ou des morts, mais aussi au silence imposé à la société, aux êtres (sur-)vivants, eux-mêmes muets comme des morts, dupliquant le silence des morts du conflit récent en Espagne, à la présence si prégnante ${ }^{10}$. On pourrait même ajouter «des conflits» car, bien que l'Espagne nait pas connu la Seconde Guerre mondiale en tant que pays belligérant, elle en a connu les échos, même (et pas si) lointains. Remarquons qu'elle n'est pas éloignée non plus, du fait de son propre conflit meurtrier, de la pensée pessimiste, de l’art marqué par la mort, qui se développent après ces guerres ayant causé la disparition de millions d'individus et, dans le conflit mondial, le cataclysme de la Shoah et des «camps de la mort», qui ne manquèrent pas d'influer sur l'art et l'écriture du monde occidental.

entera enmudezca en el tormento, / dadme un Dios para decirle lo que sufro " (source identifiée par nos soins [merci à notre collègue germaniste Helga Meise] : poème «À Werther" de 1824 publié dans sa Trilogie de la passion [Trilogie der Leidenschaft] en 1827).

8 Respectivement de 16,16, 11 et 13 poèmes, soit une répartition assez harmonieuse, destinée possiblement à tromper le chaos de la mort qui s'approche, mais sans doute en vain, vu le mauvais fatum qui s'annonce avec la dernière partie, constituée, quant à elle, du chiffre fatal de 13 poèmes. Ces parties sont d'ordinaire identifiées et synthétisées comme suit par la critique, à bon droit: rencontre du poète avec la mort; rencontre avec Dieu; acceptation résignée et souffrante de son destin; enfin, retour solitaire à la vie et lutte avec la tristesse.

9 «Tiempo aquel de gran silencio [...], tiempo de gran mutismo que sucedió al estruendo, a la metralla y al terror de los españoles en guerra [...]». Parmi ces morts du recueil, on retrouverait de manière latente des poètes républicains morts ou même exilés pendant la guerre, selon Julio Maruri, qui cite nommément García Lorca ou Antonio Machado, hypothèse crédible à lire dans la préface de Julio Maruri à A. García Cantalapiedra (García Cantalapiedra 1975: 15-16).

10 «Los muertos son seres reales, idealizados en ese plural de conjunto [...]» écrit avec justesse Gonzalo Sobejano dès 1948 dans son étude pionnière reprise dans un autre ouvrage - riche, quant à lui, de sa compilation d'articles critiques précoces sur notre poète - d’A. García Cantalapiedra (García Cantalapiedra 1971: 135). 
L'euphorie de la paix mondiale - un instant retrouvée après les crises et les conflits - est de très courte durée, immédiatement suivie d'une Guerre Froide entre les deux «blocs» idéologiques; et celle, totalement artificielle, du nouvel État espagnol n'incite guère à des productions allègres où l'homme aurait confiance en son prochain. Mais en Espagne, à l'époque, on ne doit pas le dire... Hidalgo joue alors avec un pessimisme métaphysique très général pour signifier le malheur qui pèse sur lui et parfois sur les siens: "Silencio sobre el mundo $[\ldots] »$ dit ainsi le tout premier vers du recueil, allant jusquà évoquer un «[...] cielo caído».

Et même si la voix poétique, indéterminée du point de vue des «personnes du verbe», dit ensuite que "Dios es sobre vosotros [...]» et loue ainsi Sa grandeur (et Sa pesanteur...), la suite de ce vers est ambiguë: «Azul tiene su carne, / azul su vasta sangre inmensamente lúcida: / azul es el silencio del mundo [...]». Il faudrait en effet relever - ce qui a été insuffisamment fait par les commentateurs antérieurs, nous semble-t-il - l'allusion codée à la couleur bleue, c'est-à-dire la couleur de la droite et du camp conservateur en politique ${ }^{11}$, d'autant que le sémantisme de cette couleur, répétée de nombreuses fois tout au long du recueil, est très souvent le même chez Hidalgo, à polarité mortifère ou dysphorique, exactement comme ici. Par ailleurs, il y a bien peu de couleurs dans ce recueil, entre noir et gris, comme si le poète se faisait l'apôtre du chromoclasme le plus extrême pour restituer cette ambiance sombre.

De plus, la crise du silence est bien celle du poète, ou de tout autre «témoin » ${ }^{12}$ qui veut parler aux autres, ce qui revient au même après une telle catastrophe, car la poésie (c'est-à-dire les «jolis mots», les belles phrases ou expressions) ne peut plus sourdre de la même façon, tout comme le concept de Dieu a du mal à perdurer au lendemain de la Seconde Guerre mondiale ${ }^{13}$. D'où un vers comme: « ¿Cantar?... ¿cantar?... ¿Quién canta? [...] // Hay almas, pero callan» (97): derrière le silence des morts, ce sont les vivants qui sont contaminés par cette impossibilité de dire, d'abord parce qu'il y a de l'indicible (de la douleur), ensuite et enfin parce qu'il ne faut surtout pas oublier qu'il y a également de la censure ${ }^{14}$.

Dans le poème "Los amigos muertos", on lit peut-être une évocation cryptée d'amis disparus à cause de la guerre - on sait que les sympathies de

11 Par opposition au rouge, même s'il y a de notables exceptions. Mais, de la couleur du manteau de la sainte Vierge, dans la religion chrétienne, aux codes couleur des partis conservateurs européens, dont le Partido Popular, en passant par la couleur de la royauté française et celle de la "División Azul», pour prendre des exemples dans une grande variété d'espaces et de temps, le bleu est d'ordinaire lié à la droite politique et au conservatisme idéologique.

12 De plus, «témoin [de Dieu] » se dit, en grec ancien, «martur», qui a donné «martyr».

13 Voir par exemple Le concept de Dieu après Auschwitz (Der Gottesbegriff nach Auschwitz) de Hans Jonas (1987).

14 D'autant, aussi, que derrière le verbe "cantar», on sous-entend l'expression d'un certain dynamisme, d'un élan, d'un allant, sinon d'une joie, qui ne peuvent désormais plus se faire entendre. 
José Luis Hidalgo allaient aux Républicains, même s'il ne s'est pas engagé plus avant de leur côté -, écrite à la première personne, comme les autres poèmes du recueil désormais. Ce qui est une manière, comme nous l'annoncions, de lier l'expression de l'intime à un désastre collectif, lisible ainsi par le lecteur complice et/ou éveillé, sans que le censeur puisse y trouver à redire stricto sensu... sauf à censurer alors le recueil tout entier!

Le je poétique peut alors regretter sous forme de prosopopée la disparition de ses chers amis, "[a]unque [él mismo] [s]e hunda, aunque [s]e arranque / hasta la sangre [s]e golpee», ce qui montre le côté irrémédiable de cet état des choses, doublé d'un oubli pernicieux, dont la syntaxe de la phrase qui l'exprime relie subtilement le personnel et le "groupal», en passant insensiblement de l'un à l'autre: «Solo; en la noche, yo os recuerdo / y hasta el recuerdo se desvanece». Un élément supplémentaire à retenir, dans le dernier quatrain, serait la gêne ressentie par le personnage poétique à rester vivant sur cette terre désolée, entre T. S. Eliot et José Hierro ${ }^{15}$ : «Y me avergüenzo de este cuerpo / que con los vivos me sostiene» (107).

La présence de Dieu, comme chez le Dámaso Alonso de Hijos de la ira trois ans auparavant ou dans l'œuvre de la première posguerra de José Hierro, est très problématique dans ce recueil. Sans aller jusqu'à dire qu'elle est purement rhétorique, comme chez certains poètes non franquistes de l'époque, obligés, en quelque sorte, de donner des gages à la censure, elle pose en effet plusieurs questions. Dans le dernier poème de la première partie, par exemple, intitulé "Amanecer", il semble y avoir un certain optimisme dans cette manière qu'a le sujet poétique - en s'adressant à Dieu à la fin du poème, via son « [a]gua de siglos [...]» - de s'abandonner à Lui, qui le sauverait ainsi de la mort : "Cuando los ojos de Dios se abren / amaneciendo sobre la tierra, // yo abro los míos para todo / y en todo veo Su belleza ${ }^{16}{ }$.

Malgré tout, dans ce même poème, on lit des expressions propres aux grands mystiques - notamment espagnols - qui nous font pourtant douter du vitalisme retrouvé du locuteur poétique: en effet, dans "y una luz pura me traspasa / y como un agua azul me anega», les deux verbes soulignés par nos soins, quoique baroques et empreints, on le répète, d'une extase mystique topique, équivalent à une nouvelle mort du sujet poétique, violente, qui plus est ${ }^{17}$. Le recours à l' "anéantissement» mystique, comme chez sainte Thérèse ou saint Jean de la Croix, semble en réalité fort commode pour «faire passer» - dans les deux sens

15 Respectivement dans La terre vaine/gaste/dévastée, selon les traductions françaises (original: The Waste Land), de 1922 et Tierra sin nosotros, cité plus haut, de 1947.

16 On notera néanmoins des variations dans le choix du poète de mettre ou non des majuscules aux pronoms se référant à Dieu, ce qui montre à tout le moins une hésitation quant au degré de majesté à Lui conférer réellement.

17 Et l'on ne relève même pas cette nouvelle occurrence du «bleu» qui va dans le même sens idéologique que précédemment. 
du terme - cette disparition d'un personnage poétique pourfendu puis comme «noyé» et renvoyé à son néant.

Le poème se conclut d'ailleurs sur un dernier vers ô combien ambigu et signe de crise, cette fois religieuse, même si elle est ici explicitement personnelle. Ce qui ne l'empêche pas dêtre une prise de parole risquée en temps de puritanisme national-catholique où, bien évidemment, le sujet poétique ne peut pas encore dire «nous", mais peut user d'une formule à valeur incitative pour le lecteur, à savoir celle de «douter» de Dieu: "y no comprendo que no muera [yo] », qui se réfère à la fois au miracle d'une survie et... à la totale incompréhension de cette survie $^{18}$ (112). Ce poème est, enfin, une transition vers la deuxième partie, elle aussi ambiguë quant à la vision de Dieu qu'elle va proposer.

Dans la deuxième partie, le poème intitulé «El sueño de Dios», de la même manière que le poème précédemment évoqué, souffle le chaud et le froid dans cette nouvelle prosopopée partielle qui prend son cadre dans un «[c]eleste azul [où vit] un ángel sombrío». Cet «ange sombre» à l'identité incertaine, que le poète laisse deviner par le lecteur espagnol de poésie des après-guerre(s), «ha incendiado la estrella del poniente", avec des répercussions que ce choix d'un passé composé (souligné ici par nous) restitue pleinement.

Et bien que "[a]quí en la tierra, donde el hombre habita, / ha doblado el terror su negra frente», dans un discours très générique et flou, les vers suivants montrent bel et bien un Dieu endormi: «Duerme, duerme el Señor, duerme sin ojos / en la noche total que lo sostiene»: Dieu devient alors un dormeur sans yeux, donc aveugle, dans une obscurité complète et symbolique par/dans laquelle, de plus, Il se maintient. Le compliment à Dieu - dont on évoque le «rêve» ou le «sommeil», donc - est pour le moins sujet à caution.

Une analyse des autres termes, de leur agencement ou même de la syntaxe, amphibologique («Tus hondos ojos desde mí te miran, / un sombrío velar, hasta la muerte», avec la mort, de plus, comme dernier mot du poème, disant par conséquent son retour), prouverait à l'envi ce que nous avançons quant au doute porté sur la figure de Dieu et Son dessein réel pour les hommes comme pour le locuteur, tous en situation particulièrement critique.

En effet, dans ce poème, se conjuguent à la fois le je («[...] voy por el sueño», premier vers), le "vous» du vosotros/amis lecteurs et frères humains («Podéis amar $[\ldots] »)$ et le retour au je des vers finaux: «[...] desde mí $[\ldots] »$, en passant par le «tu» divin («Tus hondos ojos [...]») et l'impersonnalisation du discours général («[...] en la tierra, donde el hombre habita» [119]), soit un bel exemple - parmi bien d'autres - de cette imbrication des voix qui parlent ou auxquelles on s'adresse, conjuguant subtilement le singulier et le pluriel.

18 Avec une autre lecture possible, davantage inscrite dans la Doxa: comment ne pas mourir d'extase face à l'amour de ce Dieu? Comme à chaque fois, l'une n'exclut pas l'autre pour autant. 
Dans «Ahora que ya estoy solo ${ }^{19}$, premier poème de la déréliction de cette troisième partie, le je poétique - qui insiste sur son extrême solitude (existentielle et métaphysique? circonstancielle et historique? un mixte des deux, sans doute, comme on l'a compris au vu de cette stratégie d'écriture de crise) - s'adresse à (un) Dieu. Ce Dieu nest, pas plus ici qu'ailleurs, identifié au Dieu nationalcatholique avec Ses attributions canoniques austères et justicières. Le locuteur poétique L'appelle afin qu'Il l'accompagne jusqu'à la mort, ou, pour mieux dire, qu'Il fasse venir la mort, puisque dans ce poème Dieu est la mort. Derrière cette vision digne de tout croyant, certes - car, qui voit Dieu voit la mort, et vice versa -, on lit une présentation de cet état de fait par le biais d'une équivalence si logique et si résignée qu'elle en a quelque chose de pesant: «[...] Tú sabes / que a la muerte hay que ir sin que nadie nos llore». Langoisse possible du lecteur est d'autant plus forte que l'image récurrente de l'arrivée de Dieu/la mort retenue dans ce cas-ci par le poète, c'est-à-dire celle d'un fruit, fait immanquablement penser à un fruit empoisonné, nouvelle pomme du Paradis perdu qui précipitera le locuteur solitaire dans le sommeil éternel: «y bajas a mi boca como un fruto maduro ». Le distique final, résigné à la volonté divine qui lui intime de croquer le fruit empoisonné, insiste sur cette acceptation de la fatalité: «Por eso está ya el fruto temblando entre mis dientes, / mas no quiero morderlo sin que Tú me lo digas» (131).

Bien entendu, du fait de ces non-dits systématisés, cette lecture n'est pas la seule possible, et l'acceptation des desseins de Dieu est bien l'une des règles de base de tout croyant, ce que la censure ne pouvait condamner, mais Hidalgo joue précisément avec cette idée pour transmettre une autre version moins "catholique» du rapport à un Dieu quelque peu désabusé et velléitaire, vu ici comme une Parque insensible. Enfin, l'usage d'un "nous» furtif fait passer, presque sans que l'on s'en rende compte, un propos d'ordre éminemment personnel à une vérité d’ordre plus général qui, si elle ne ressortit pas non plus à l'amplitude de l'Histoire même ou d'un lieu précis ${ }^{20}$, rend le propos collectif, donc la possibilité d'identification du lecteur plus aisée : «que a la muerte hay $q u e^{21}$ ir sin que nadie nos llore, / ocultando las rosas que encendimos» (131) (nous soulignons).

19 Début du premier vers ( Ahora que ya estoy solo te llamo suavemente»), donc début éponyme du poème, et expression que l'on retrouve au début du second vers («Ahora que ya estoy solo puedo morir [...]»), d'où son importance, renforcée par le "puedo morir» qui programme un destin fatal bien précis et très précoce, totalement accepté ici par le je poétique.

20 Nous voulons signifier par là que le poète ne parle pas ici précisément de l'Espagne ni de la posguerra, comme il ne le fait d'ailleurs jamais dans son recueil, par impossibilité totale de le faire à cause de la censure, ce qui est un trait de la poésie «desarraigada » d'époque.

21 Dans ce cas-ci, le discours général est initialement rendu par le biais, non pas d'une première personne du pluriel, mais d'une formule d'obligation impersonnelle, qui trouve son prolongement, on le voit, dans la même phrase, par deux «nous» alors identiques dans leur portée à ce «il faut» qu'ils confirment. 
Dans le poème «Tristeza », premier poème de la quatrième et dernière partie, au titre lui aussi programmatique d'un certain désespoir et d'une situation de malheur sans futur riant en vue, on en revient à une mélancolie, une nostalgie, un état de malaise brut. Dieu semble oublié, dans un paysage terrestre figé («tierra inmutable $[. .]$.$» mais en même temps "poderosa», comme l'Espagne de Franco$ et ses rêves d'Empire retrouvé?) et céleste gagné, quant à lui par la paix («cielo sereno $[\ldots] »)$, même si l'on sait le sémantisme second de cette "sérénité» chez certains poètes de l'après-guerre espagnole: en effet, la "paix» imposée était souvent synonyme latent et implicite, chez bon nombre de poètes ibériques, de «mort clinique» du peuple et du pays, comme pris dans la glace de cette fausse trêve spécieuse et forcée, où rien n'est résolu, au fond, entre les «deux Espagnes».

La temporalité reste vague en apparence («[...] donde el tiempo / pasa ${ }^{22}$ lejano y nunca mira...»), mais est à relier en quelque sorte à cet espace ("pasa lejano", expression "spatiale» très étrange pour évoquer du temps) et à une situation critique, même à un désastre, chronologiquement passés, mais encore proches dans le ressenti, avec ces «flores ocultas de ceniza». Ladjectif clé est bien ce «ocultas», mise en abyme du sous-texte idéologique et/ou social des stratégies poétiques proposées par Hidalgo. Enfin, la référence à un «amor vago» (143), répété dans deux vers, est une autre mise en abyme possible, pour le lecteur, de ces évocations et allusions délibérément floues d'un état des choses sur lequel, du fait de la censure, l'écrivain ne veut, ne doit ni ne peut donner de nom plus précis, comme pour toute chose dans ce recueil écrit et publié sur la corde raide.

Dans «Nacimiento», avant-dernier poème, au titre a priori plus optimiste, le je poétique amalgame encore une fois le discours collectif et personnel (dans cet ordre: "Ha llegado la noche para todos: / yo reclino mi frente en esta piedra", nous soulignons), pour dire la nécessité d' «ouvrir les yeux» afin dêtre attentif, lucide et décisionnaire en liberté de choix assumée face à sa vie personnelle - une sorte d'existentialisme déguisé - et d'éviter ainsi la paix délétère de la passivité, de la léthargie, voire du sommeil létal; c'est-à-dire cette aboulie lénifiante du non désir, de la non action, donc de l'état de mort-vivant que l'on a évoqués plus haut comme caractérisant l'Espagne des années quarante.

Par conséquent, l'espace d'un instant, le je poétique peut (se et nous) dire: «Ni me muero, ni sueño ${ }^{23}$. Abro los ojos / y extendiendo mis manos verdaderas / toco el origen de mi ser humano» (154), bien que Dieu soit là encore le "dernier mot», le «maître mot», in fine (comme au début...) de tout être et de

22 Dans le même poème est répété le verbe "pasar ", plus loin, dans le sens, dans ce cas, de «s'en aller»- même si plausiblement polysémique aussi -: «[...] Pero pasan / todas las cosas, alma mía, / como las nubes y las rosas / pasan, pasan... [...]». L’idée d'une volatilité et d'une caducité rapides de toutes choses est certaine.

23 On l'a compris, le «sueño» est l'envers de la vie, même si celle-ci est douloureuse, rude et pleine d'aspérités. Ce «sueño», ici première personne du présent de l'indicatif du verbe «soñar», représente bien la mort, très classiquement. 
toute chose ${ }^{24}$. Mais au moins le sujet poétique vit et prend conscience (quasi sartrienne) de son être par son corps, sa source d'existence.

Le dernier poème, un romance, mais ennéasyllabique ${ }^{25}$ (d'où une certaine liberté vis-à-vis du canon), s'intitule «Belleza» et clôt donc le propos poétique de José Luis Hidalgo. Il subsume les paradoxes, contradictions et ambiguïtés que l'on a pu déceler tout au long de son recueil, en confirmant également une lecture pessimiste certaine du monde, malgré les éclairs d’optimisme que l’on sent quelque peu obligés et qui zèbrent de manière plus quéphémère le ciel noir de ce recueil.

Derrière ce titre, la beauté des choses est montrée comme brûlante, invisible, dans la nuit (laquelle? historique ou existentielle? les deux, là encore, sans doute). Mais, très vite, «[...] la ceniza se derrama / sobre el silencio de su ser [de las cosas]», les sifflantes du silence épaississant ledit silence, augmenté d'une couche de cendre, terme que l’on a déjà rencontré et qui se réfère à la mort, la mort froidie, mais encore présente. De même, le Dieu évoqué dans Los muertos apparaît dans cet ultime poème comme "[...] oculto [...]», et la trace de Son pied est volatile, car «[...] efímera se apaga / cuando brota el amanecer». Elle disparait quand les hommes, eux, se lèvent pour vivre leur journée, c'est-à-dire leur vie, c'est-à-dire au moment où ils en auraient le plus besoin...

Hidalgo peut alors donner à lire au lecteur un dernier quatrain métapoétique ${ }^{26}$ qu'il vaut la peine de commenter: mettant en exergue sa qualité de poète - avec article défini et majuscule de rigueur -, le je poétique dit à la fois son essence privilégiée et responsable de témoin et de "passeur» pour une communauté27, mais aussi son incapacité finale à établir, donc à dire, la nature de ce lien avec Dieu et avec les choses.

En effet, cette beauté - naturelle, donc divine - s'évanouit au petit matin, et le poète n'a plus que ses mains qui peinent à prendre la plume pour dire cet indicible, car incompréhensible, insaisissable. Cette fin de poème, et de recueil, est certes plus métaphysique que politique. Mais ce doute quant à la capacité du poète à transmettre au lecteur frère ce qu'il y a d'indicible dans le monde soient les rapports très particuliers à un Dieu dont la présence est pour le moins intermittente - et ce, à une époque profondément troublée, reflète une crise profonde qui englobe et le je et le tu du lecteur, un «nous» sous-entendu dont la

24 Il faudrait étudier également le rapport d'Hidalgo à la nature et aux éléments, dans ce recueil, ce que nous n'avons pas la place de faire.

25 Sur la métrique du recueil, on notera simplement qu'il y a plus souvent des endécasyllabes à la Bécquer, répartis en quatrains (et parfois en sizains) que des ennéasyllabes (et parfois des alexandrins), mais que tous ces vers sont répartis sous forme de romance, ce qui montre un double respect à la fois envers la tradition poétique et la forme populaire, et l'ouverture à une certaine modernité.

26 «Soy el Poeta. Me pregunto: / ¿qué es lo que anoche sentí arder? / Miro mis manos trastornado / y no lo puedo comprender» (153).

27 N'oublions pas que, un peu plus haut dans le poème, on peut lire un «nous» collectivisant: «El Dios oculto que nos vela». 
portée est autant sociale qu'existentielle et qui va bien au-delà de la petite intimité du seul locuteur poétique.

En conclusion, on peut dire que José Luis Hidalgo, en tant que poète espagnol en proie à la crise d'une époque et d'un pays, dans cette première posguerra, et tout comme les autres poètes non affiliés notoirement au Régime, mais sans en être non plus de ses contempteurs extrêmes, ne pouvait guère critiquer avec précision un pays et une société, et se devait de passer par une critique vague et floue "d'un» monde qui l'est tout autant. Une critique traduite par des doutes existentiels - mais pas tout à fait existentialistes, on a dit pourquoi - qui étaient bien réels, d’ailleurs, pour le poète, et où Dieu, s'il est interrogé et parfois incriminé par le je poétique, au moins "existait» Lui aussi, ce qui suffisait provisoirement au bonheur de la censure... La mort étant un thème clé de la poésie depuis ses origines, cette écriture, pour pessimiste qu'elle fût, était acceptable et partageable par tous, ce qui constituait un expédient pratique pour s'adresser en sous-main au plus grand nombre, d'autant que l'époque, en Espagne et ailleurs - de manière plus ou moins explicite, bien entendu -, était davantage à l'obsession morbide qu'au plein optimisme.

Il importe alors peu que, à l'origine, notre poète ait souhaité parler effectivement des morts de la guerre civile espagnole, ce qui compte, c'est que cette transformation soit allée, pour des raisons de contournement de la censure et / ou d'élargissement du propos, vers une écriture d'universalisation qui la rend terriblement permanente.

On a vu aussi que, dans la stratégie d'écriture de José Luis Hidalgo, entre ambiguïtés et polysémie, le jeu d'aller-retour entre la crise de l'intime et celle du collectif était un recours qui permettait de relier entre elles l'expression du moi lyrique et la prise de conscience possible d'un «nous» proche de lui, jusqu'au lectorat complice qui recollait en dernier lieu les pièces du puzzle langagier en le décryptant comme étant davantage d’ordre social ou idéologique. C’est peutêtre une bien maigre consolation que de voir un poète, face à cette crise du signe poétique provoquée par la crise "réelle» du temps, s'atteler à ce type de stratégie de l'allusion et du «double-entendre" pour se faire lire. Mais cétait bien la seule possible alors, et cette stratégie a aussi, reconnaissons-le, une portée universelle et pas seulement historique, de par son fond existentiel, justement, qui nous touche et nous concerne tous. Sinon, on ne lirait plus aujourd'hui cette poésie.

Différentes lectures - autorisées, d'ailleurs, par le médium poétique lui-même, par essence amphibologique ${ }^{28}$ - sont donc possibles, et les Vainqueurs aussi ont

28 D'où les mots de José Hierro dans son «Aniversario José Luis Hidalgo» (Alerta, Santander, 5 février 1950), à propos du recueil de son cadet, et qui s'y connaissait en langage poétique faussement lisse, vraiment polysémique et jouant constamment sur la suggestion, comme Hidalgo: «Debía hablaros de cómo Los muertos [...] recoge todo el misterio, la incertidumbre, la angustia de una época. [...] [Pero] [y]o no sé qué deciros. Hoy [en 1950] hay que contar, no evocar con vaguedades, con lirismos, con oscuridades trasnochadas. Los tiempos mandan. [...] Como si no fuera una 
pu apprécier, à l'époque, ces vers parfois mystérieux de Hidalgo, en insistant précisément sur la souffrance métaphysique d'un homme qui cherche Dieu. Le sens final précis de ces poèmes est donc, au-delà de l'acte créatif et solitaire, indécidable, car son ambiguité est "programmée» ab ovo pour le lecteur, qui seul peut trancher dans cette négociation permanente entre ce qui lui est donné à lire et ce qu'il veut en extraire in fine. Une ambiguïté et de l'indécidable encore renforcés par les stratégies d'écriture en période de censure dont nous avons parlé. La plupart des commentateurs évaluent et louent ainsi les deux versants, tout à la fois l'intime - par lecture première - et l'historique - par induction -, de cette ouvre ${ }^{29}$.

En repensant au terme de "crise» comme l'instant où tout pourrait changer, aussi et peut-être, «en bien», nous souhaitons, personnellement, achever cette réflexion en prenant davantage parti et en insistant sur la nécessité de rappeler la source incarnée, concrète et «historique» de la douleur chez Hidalgo avant sa transmutation en langage maîtrisé, transmissible au groupe et approchant à la fois l'âme, l'abstraction et l'éternité:

Aquí denunciaremosun error: se equivocaría quien pensase que tú [Maruri s'adresse à l'ami poète mort] "poetizabas» desde un espejismo ahistórico, un lugar ideal donde la temporalidad del ser del hombre no tuviese otra realidad que la de servir de soporte al cántico [...]. Tú, sonriente y lúcido, cantabas la realidad de la calle en pesetas y mano de obra $[\ldots] . .^{30}$

En cela, le dernier poème du recueil, «Belleza», étudié plus haut, nous semble paradigmatique de la poétique de distanciation par Hidalgo de son je lyrique, déjà lisible dans ses apostrophes et références à une collectivité. En effet, et en dépit de cette crise du signe poétique en période de précontrainte de tous ordres, cette «belleza» sonne pour nous comme la mise en forme poïétique et esthétique,

maravillosa manera de hablar esa que nada dice, que nada cuenta, aunque lo insinúe todo» (cité par García Cantalapiedra 1971: 147).

29 Par exemple, l'un des plus grands connaisseurs actuels de l'œuvre hidalguienne, J.A. González Fuentes, écrit que: «Los muertos debe leerse como el primer fruto magnífico y maduro de un aprendizaje (vital y literario) trágicamente truncado por la enfermedad: debe penetrarse como el inestimable relato de quien consiguió hacerse recuerdo de su propia vida y de su propia muerte, memoria de su propio tiempo y del fracaso personal y colectivo de toda una generación de españoles a los que la guerra civil, y la posterior dictadura, dejó desbordados para siempre por el dolor, la desilusión y la más profunda amargura", dans la préface à son édition des Poesías completas de Hidalgo (Hidalgo 2000: 13-14). Mais on lira aussi: «[...] [S] on los funerales de la guerra civil y, como todo rito fúnebre, conmemoran, no las circunstancias de la muerte, su entorno lívido y la particularidad de sus víctimas, sino la misma Muerte imaginaria con que la Humanidad, por sus poetas, conjura la angustia de lo impensable», selon J. Maruri (García Cantalapiedra 1975: 15): deux citations qui montrent la nécessaire prise de hauteur du poète par rapport à l'événement concret, qui reste la base cachée du texte, pour atteindre au concept même de Mort.

30 Toujours selon Maruri (García Cantalapiedra 1975: 16). 
précisément, de la souffrance, de l'angoisse et du pathos, sa mise à distance et sa tenue en laisse ${ }^{31}$, c'est-à-dire le seul moyen, au fond, pour un homme, le poète, d'apprivoiser la douleur, de la faire «se tenir plus tranquille».

\section{Bibliographie}

Fernández, L., 1983, Recherche sur la poésie existentielle espagnole des années quarante: approche de Dieu, de l'au-delà et de la mort dans la poésie de José Luis Hidalgo, Toulouse, Université de Toulouse II-Le Mirail (inédite).

García Cantalapiedra ,A. (ed.), 1971, Verso y prosa en torno a la obra de José Luis Hidalgo, Santander, Diputación Provincial, Institución Cultural de Cantabria, Instituto de Literatura "José María de Pereda».

García Cantalapiedra, A., 1975, Tiempo y vida de José Luis Hidalgo, Madrid, Taurus.

Gide, A., 2010 (1900), De l'influence en littérature, Paris, Allia.

Goytisolo, J., 2012, Ardores, cenizas, desmemoria, Madrid, Salto de Página.

Hidalgo, J.L., 2000 (1947), Los muertos, in: Poesías completas (ed. de J.A. González Fuentes), Barcelone, DVD; $1^{\text {re }}$ éd. Madrid, s.n. [Gráf. Urguina], «Adonais» $\mathrm{n}^{\circ}$ XXXIV.

Le Vagueresse, E., 2004, «Lire en Espagne sous Franco: l'heure du lecteur», in: Bénat-Tachot, L., Vilar, J. (dir.), La question du lecteur, Marne-la-Vallée, Ambassade d'Espagne/Presses Universitaires de Marne-la-Vallée.

Maruri, J., 1975, «Huésped de la memoria», in: García Cantalapiedra A., Tiempo y vida de José Luis Hidalgo, Taurus.

Ory, P., 2011 (2004), L'histoire culturelle, Paris, Presses Universitaires de France.

Sobejano, G., 1948, "José Luis Hidalgo, poeta de los muertos», Verbo, Alicante, novembre-décembre, repris in: García Cantalapiedra, A. (ed.), 1971, Verso y prosa en torno a la obra de José Luis Hidalgo, Santander, Diputación Provincial, Institución Cultural de Cantabria, Instituto de Cultura «José María Pereda».

31 «[...] [A]l fin [...] la evocación de la belleza misteriosa, [es] quizá la única tabla de salvación que avistó [Hidalgo] en su interior naufragio», selon Sobejano (García Cantalapiedra 1971: 134). 